\title{
I marcatori di infiammazione e coagulazione come predittori di eventi avversi severi nei pazienti HIV-positivi in terapia antiretrovirale soppressiva Inflammation and coagulation markers as predictors of severe adverse events in HIV-positive patients on successful antiretroviral treatment.
}

\begin{abstract}
Alessandra Bandera
School of Medicine and Surgery, Department of Pathophysiology and Transplantation, University of Milan; Infectious Diseases Unit, Department of Internal Medicine IRCCS Ca' Granda Ospedale Maggiore Policlinico Foundation
\end{abstract}

\section{Riassunto}

L'immunoattivazione e l'infiammazione persistono nonostante la terapia antiretrovirale soppressiva e sembrano predire molte delle morbidità che affliggono i pazienti con infezione da HIV nell'era moderna. Obiettivo di questa review è di analizzare le evidenze più recenti che suggeriscono un legame tra lo stato di infiammazione persistente e la morbidità/mortalità in questo setting. Abbiamo focalizzato la nostra attenzione sulla letteratura recente che mostra che la morbidità e mortalità associate all'attivazione dei meccanismi di infiammazione e coagulazione includono condizioni patologiche anche diverse dagli end-point clinici classicamente analizzati, quali AIDS, malattia cardio-vascolare e neoplasie non-AIDS definenti. Interventi che riducano I'infiammazione in modo efficace potrebbero avere un impatto significativo sullo stato di salute dei pazienti con HIV.

\section{Abstract}

Immune activation and inflammation persist despite suppressive antiretroviral therapy and likely predict many of the morbidities that afflict HIV-infected patients in the modern treatment era. The goal of this review is to analyze the most relevant evidence suggesting a link between the persistent inflammatory state and morbidity and mortality in this setting. We focused our attention on recent literature showing that morbidity and mortality associated with activation of inflammatory and coagulation pathways include also conditions other than AIDS, cardio-vascular disease, and non-AIDS cancer events. Effective inflammation-dampening interventions could greatly affect the health of people with HIV.

\section{Introduzione}

Grazie all'utilizzo della terapia antiretrovirale di combinazione (ART), da almeno un decennio, la morbilità e la mortalità HIV-relata hanno subito un passaggio significativo dalle patologie AIDSrelate agli eventi non-AIDS, come le neoplasie, la cirrosi epatica, l'insufficienza renale e gli eventi cardiovascolari (1).

Molteplici evidenze in letteratura suggeriscono la presenza di una precisa associazione tra lo stato di infiammazione persistente, nonostante la soppressione virologica ART-mediata, e le morbidità/mortalità attribuite a tale condizione denominate serious non-AIDS events (SNAs). Nel corso degli anni, si sono susseguite molteplici osservazioni che hanno evidenziato come alcuni marcatori di infiammazione (come IL-6, fibrinogeno, recettore solubile di tumor necrosis factor (sTNFR1), sTNFR2 e high sensitive C-reactive proteine-hsCRP), marcatori di attivazione monocitaria/macrofagica (CD14 solubile) e marcatori di disfunzione della barriera epiteliale intestinale (zonulina, intestinl fatty acid binding protein (I-FABP)) possano predire la mortalità in corso di infezione da HIV in trattamento, anche nei pazienti con conta dei linfociti T CD4+ elevata. Scopo di questo lavoro è la revisione delle conoscenze attuali sull'associazione tra i marcatori di immunoattivazione e gli eventi avversi non-AIDS relati, analizzando nello specifico i dati più recenti che suggeriscono un ruolo nell'infiammazione non solo nelle patologie cardiovascolari e oncologiche ma anche negli altri eventi avversi.
Autore per la corrispondenza:

Alessandra Bandera Infectious Diseases Unit, Department of Internal Medicine IRCCS Ca' Granda Ospedale Maggiore Policlinico Foundation Via Francesco Sforza 28, 20122 Milano

alessandra.bandera@unimi.it

Keywords:

infiammazione; coagulazione; eventi avversi.

Potenziali conflitti di interesse:

nessuno.

JHA 2018; 3(4): 77-80

DOI: 10.19198/JHA31458 
Il ruolo dei marcatori di infiammazione e coagulazione come predittori di mortalità ed eventi non-AIDS nei pazienti in terapia antiretrovirale soppressiva

II trial SMART (Strategies for Management of AntiRetroviral Therapy) ha chiaramente dimostrato che I'uso intermittente rispetto all'uso continuativo della ART si associa ad un eccesso di mortalità per qualsiasi causa, con la maggioranza dei decessi attribuibili a patologia cardiovascolare e cancro (2). Del pannello di 6 biomarcatori studiati al baseline nei partecipanti dello studio SMART, 3 marcatori si sono evidenziati come predittori indipendenti di morte durante il follow-up: IL-6, PCR ad alta sensibilità (hsCRP) e D-dimero. Due studi recenti hanno cercato di rispondere ad un quesito fondamentale, ossia se l'infiammazione generalizzata e lo stato di ipercoagulazione contribuiscano alla patogenesi di multiple morbidità o siano associate allo sviluppo di specifiche patologie.

Il primo studio (3) ha valutato i pazienti appartenenti ai bracci di controllo degli studi SMART ed ESPRIT (Evaluation of Subcutaneous Proleukin in a Randomized Internationl Trial) analizzando la correlazione dei livelli basali di IL6, hsCRP e D-dimero come predittori di differenti end-point clinici. L'analisi è stata condotta sui partecipanti ad entrambi i trial $(n=4304)$ di cui erano disponibili i livelli plasmatici di IL-6, hsCRP e D-dimero all'ingresso nello studio, prima della randomizzazione. I pazienti analizzati, appartenenti ai bracci di controllo di entrambi gli studi, ricevevano una terapia ART standard (secondo le linee guida) in modo continuativo. Sono stati considerati i seguenti end point clinici: (i) mortalità per qualunque causa; (ii) decessi per eventi non-AIDS, non violenti e non intenzionali, (iii) progressione ad AIDS (fatale e non fatale), (iv) eventi CVD (fatali e non fatali); (v) neoplasie nonAIDS definenti (fatali e non fatali). L'analisi ha confermato che il meccanismo fisiopatologico che sottende l'aumentata produzione di IL-6, hsCRP e D-dimero è diverso rispetto alle varie tipologie di eventi clinici considerati. In particolare, livelli incrementati di IL-6 al baseline hanno dimostrato un potere predittivo maggiore, rispetto a hsCRP e D-dimero, di sviluppare successivi eventi CVD e tumori non-AIDS relati, confermando dati precedenti che supportano un ruolo per $i$ polimorfismi genetici di IL-6 nell'induzione di un aumentato rischio di CVD e neoplasie. Al contrario, quando si sono analizzati gli eventi AIDS, i livelli di IL-6 e D-dimero sono risultati similmente associati ad un aumentato rischio di progressione ad AIDS, sottendendo un ruolo potenziale di D-dimero nella patogenesi delle patologie opportunistiche, da approfondire in futuri studi.

Nel secondo studio (4) è stato sviluppato uno score di biomarcatori denominato "IL-6 \& D-dimer score" che combina i marker di infiammazione e coagulazione per predire il rischio di eventi severi non-AIDS definenti o morte per qualunque causa (SNA/death). Lo studio è stato condotto su 3766 pazienti appartenenti ai bracci di controllo di 3 studi: SMART (1748 pazienti), ESPRIT (1446 pazienti), SILCAAT (Subcutaneous Recombinant, Human Interleukin-2 in HIV-Infected Patients with Low CD4+ Counts under Active Antiretroviral Therapy, 572 pazienti), restringendo I'analisi ai pazienti in trattamento ART con soppressione virologica. L'outcome principale è stato definito come SNA/death, includendo un endpoint composito costituito da malattia CVD, insufficienza renale terminale, cirrosi epatica scompensata e morte per qualunque causa. In questa analisi, è stato dimostrato che una singola misurazione di IL6, D-dimero o hsPCR al baseline predice il rischio di SNA/death e che in un modello unificato la combinazione dei livelli di II-6 e D-dimero predice in modo independente il rischio di SNA/death.

Considerato che molte delle morti registrate negli studi sopracitati non sono attribuibili ad eventi AIDS o a eventi severi non-AIDS definenti riconducibili a patologie CVD o neoplasie, ci si chiede se egli altri eventi avversi severi possano ugualmente essere ricondotti ad uno stato di aumentata infiammazione e coagulazione.

\section{II ruolo dei marcatori di infiammazione e coaugulazione come predittori di eventi avversi severi non correlati al rischio cardiovascolare o alle neoplasie non-AIDS}

Sulla scorta dei dati già citati, un lavoro recentemente pubblicato su JAIDS (5) ha valutato se la componente infiammatoria dimostrata dai livelli periferici di IL-6 e D-dimero si correli all'insorgenza di eventi potenzialmente mortali non attribuibili a AIDS, CVD e neoplasie non-AIDS relate. L'analisi ha incluso i soggetti dei bracci di 


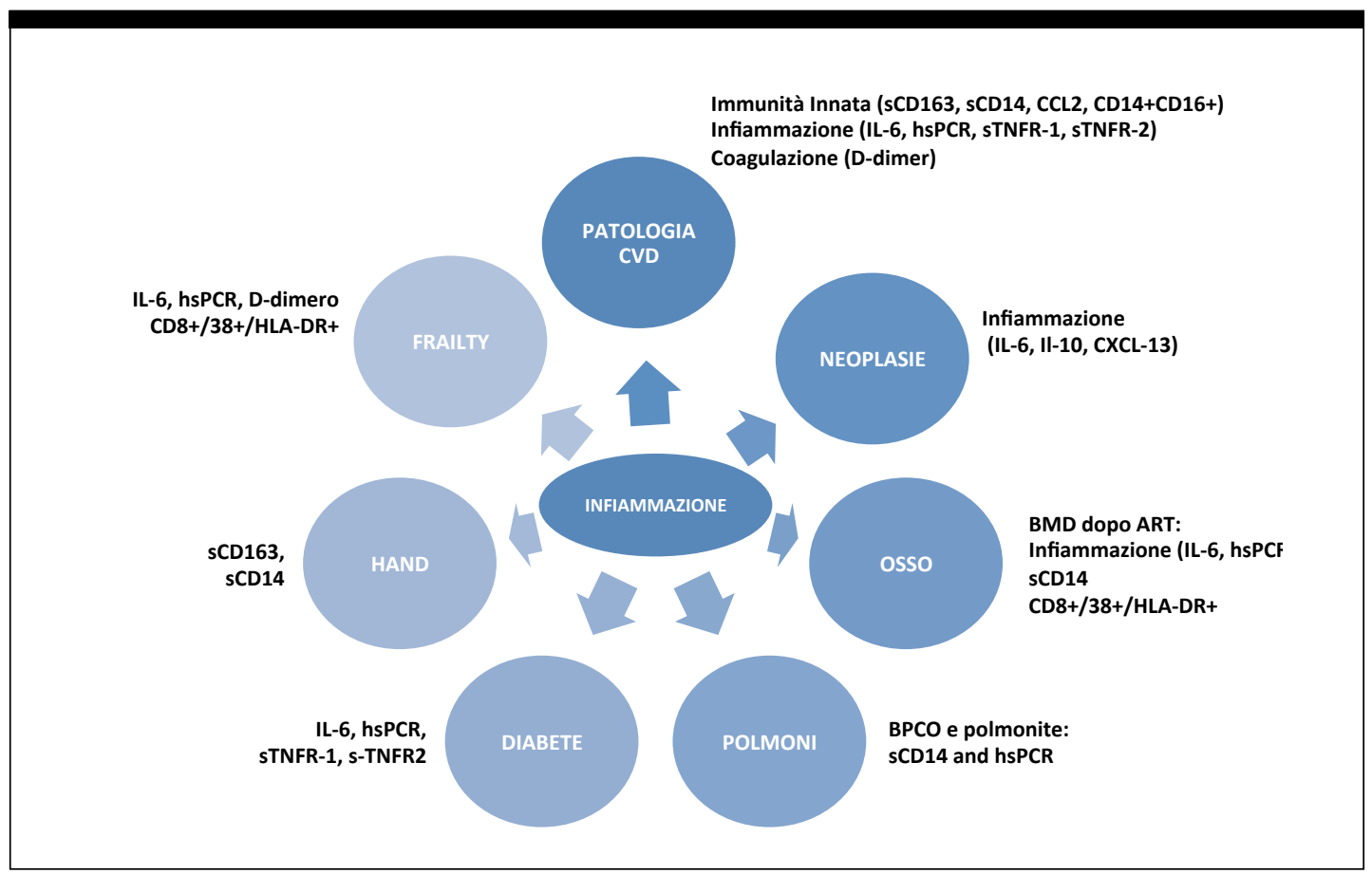

Figura 1. Associazione tra infiammazione e end-organ disease.

controllo dei medesimi trial clinici, SMART ( $n=$ 2752) e ESPRIT ( $n=2040)$ restringendo l'analisi ai pazienti in terapia ART con HIV-RNA inferiore a $500 \mathrm{cp} / \mathrm{ml}$ e CD4+ superiori a 350/mmc $(n=3568)$. Gli eventi avversi registrati nei 2 trial sono stati classificati secondo la scala di severità a 4 punti usando la tabella di tossicità della National Institute of Allergy and Infectious Diseases (NIAID) Division of AIDS. Per definire inoltre delle categorie di eventi mutuamente esclusive sono stati definiti 4 diversi outcome: (i) aventi AIDS fatali e non fatali; (ii) malattia CVD fatale e non fatale; (iii) neoplasie non-AIDS definenti fatali e non fatali; (iv) eventi di grado 4 o morti correlate ad eventi non inclusi nelle precedenti categorie. Gli eventi di grado 4 appartenenti alla quarta categoria sono stati inoltre suddivisi in 2 gruppi secondo una classificazione proposta recentemente dallo studio MESA (MultiEthnic Study of Atherosclerosis) (6) che definisce tra le patologie infettive, endocrinologiche, nutrizionali, metaboliche, del sistema nervoso, respiratorio e digestivo, le patologie cutanee, muscolo-scheletriche e del tessuto connettivo e i disordini ematologici quelle considerate correlate ad una componente infiammatoria cronica come ChIRD (chronic inflammation-related disease).
L'incidenza di eventi avversi di grado 4 (22.9 per 1000 persone/anno) è risultata 3-6 volte più elevata rispetto a quella di AIDS, eventi CVD e neoplasie non-AIDS relate. Gli eventi avversi di grado 4 ChrIRD rappresentavano il $49 \%$ degli eventi di grado 4 e tra questi i più comuni erano gastroenterite, cirrosi epatica, insufficienza renale acuta, pancreatite acuta. Tra gli eventi avversi di grado 4 non-ChrIRD i più frequenti erano rappresentati da depressione, dolore lombare, ernia inguinale e tentativo di suicidio. I livelli basali d II-6 e D-dimero, misurati anche anni prima l'insorgenza dell'evento avverso, sono risultati fortemente associati agli eventi avversi di grado 4, con una forza di associazione simile a quella dimostrata per gli eventi CVD e le neoplasie non-AIDS. In particolare l'associazione tra i livelli basali di D-dimero e il rischio di sviluppare eventi di grado 4 era analoga per gli eventi ChIRD e non-ChIRD, mentre gli eventi ChIRD si associavano ai livelli più alti di IL- 6 basale in maniera più significativa rispetto agli eventi nonChIRD. Questa analisi mette in luce dunque un ruolo dell'infiammazione residua in corso di ART virologicamente efficace nella patogenesi di uno spettro di morbidità più ampio di quanto finora ipotizzato e considerato (Figura 1). 


\section{review}

\section{Conclusioni}

Nonostante siano necessari studi più approfonditi sulle conseguenze dell'infiammazione cronica in corso di ART, sembra chiaro, tuttavia, che esistono una serie di patologie diverse dagli eventi non-AIDS finora considerati, correlate ad aumentato rischio di morte, che possono influenzare la salute dei pazienti con HIV e che potrebbero rappresentare il target di trial interventistici finalizzati a ridurre l'infiammazione persistente.

\section{BIBLIOGRAFIA}

1. Phillips AN, Neaton J, Lundgren JD. The role of HIV in serious diseases other than AIDS. AIDS. 2008; 22:2409-18.

2. El-Sadr WM, Lundgren JD, Neaton JD, et al. CD4+ count-guided interruption of antiretroviral treatment. N Engl J Med 2006; 355: 2283-96.

3. Borges ÁH, O'Connor JL, Phillips AN, et al. Interleukin 6 is a stronger predictor of clinical events than high-sensitivity C-Reactive protein or D- dimer during HIV infection. J Infect Dis. 2016; 214: 408-16.

4. Grund B, Baker JV, Deeks SG, et al. Relevance of Interleukin-6 and D- dimer for serious non-AIDS morbidity and death among HIVpositive adults on suppressive antiretroviral therapy. PLoS One. 2016; 11: e0155100

5. Hart BB, Nordell, AD, Okulicz JF,et al. Inflammation-Related Morbidity and Mortality Among HIV-Positive Adults: How Extensive Is It? J Acquir Immune Defic Syndr 2018; 77: 1-7

6. Duprez DA, Otvos J, Tracy RP, et al. High-density lipoprotein subclasses and noncardiovascular, noncancer chronic inflammatoryrelated events versus cardiovascular events: the multi-ethnic study of Atherosclerosis. J Am Heart Assoc. 2015; 4: e002295. 